\title{
Open Calculus: A Free Online Learning Environment
}

Jane Korey, (E-mail: jane.korey@dartmouth.edu), Dartmouth College Kim Rheinlander, (E-mail: kim.rheinlander@dartmouth.edu), Dartmouth College Dorothy Wallace, (E-mail: Dorothy.Wallace@dartmouth.edu), Dartmouth College

\begin{abstract}
Dartmouth College mathematicians have developed a free online calculus course called "Open Calculus." Open Calculus is an exportable distance-learning/self-study environment for learning calculus including written text, nearly 4000 online homework problems and instructional videos. The paper recounts the evaluation of course elements since 2000 in two experimental/control course situations involving nearly 300 students.
\end{abstract}

\section{INTRODUCTION}

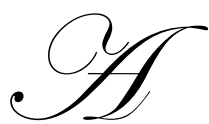

$t$ the $K-12$ level, all students need access to educational experiences that create high levels of scientific, mathematical, and technological literacy. (National Council of Teachers of Mathematics, 1989; National Research Council, National Committee on Science Education Standards and Assessment, 1995). "From Analysis to Action," Center for Science, Mathematics, and Engineering Education, National Research Council, National Academy Press, 1996

Online learning is a rapidly growing component of the educational enterprise. In the past twenty years computers have become almost as commonplace in mathematics courses as pencils and online calculus courses have proliferated on the internet. Computer-assisted mathematics learning appears to be unconstrained by cultural boundaries: the very existence of the International Journal of Computers for Mathematical Learning (one among many journals devoted to the educational uses of computers) speaks to the worldwide acceptance of computers as tools for learning math. The internet thus seems the appropriate location to address issues of educational access across subjects, levels and regions.

Open Calculus, the online learning environment developed by Dartmouth College mathematicians, is different from other online calculus offerings in two important ways: it is open source and it is free, requiring no course staff, graders or textbook. The goal of this effort is to provide free high-quality calculus instruction to anyone, anywhere, with an internet connection. The intent is for an institution to install these materials and serve their local constituency, either as a center for distance learning of calculus, as a vehicle for self-study outside a formal course or as a supplement to a standard course. Being open source, these materials can be reorganized or augmented to suit local needs. Instructors can easily upload their own course notes and add problems. Thus, although the website is in English, it is may be adapted by developers to work in any language.

The populations that stand to benefit most from Open Calculus are secondary school students in rural or underserved areas who are taking calculus or preparing for Advanced Placement examinations, undergraduates in two-year or four-year colleges, distance learning students, and working adult students who must do the majority of their studying at home. According to a position paper on the No Child Left Behind Act, published online by the National Rural Education association, less than 25 percent of schools can meet the NCLB requirement of having "highly qualified" teachers in all classrooms [1]. Yet the National Center for Educational Statistics reports that, as of 2001, 100 percent of rural schools have internet access [2]. By using online video tutorials and lectures that are keyed to a large and ever increasing collection of practice problems, Open Calculus can put a qualified calculus teacher in any classroom. 
Secondary school students can use Open Calculus to prepare for the Advanced Placement examination in calculus or to strengthen or review their high school calculus learning. The Advanced Placement Calculus Exam is a recognized benchmark of achievement for high school students in the United States and an admission criterion for many colleges and universities. Advanced Placement Calculus serves as the "gatekeeper" to higher education in math, science and engineering. Secondary school students often face significant barriers to undertaking, completing and being successful at calculus; budgetary, scheduling and staff constraints prevent many high schools from offering calculus, especially those in rural or under-funded areas. Electronic access to quality calculus instruction will help level the playing field between wealthy suburban schools and poorer institutions in inner city and rural environments. Students who have taken calculus but performed poorly on Advanced Placement tests can use this course of study to prepare for placement exams at Dartmouth or any other institution.

Two-year colleges often have non-traditional students who face special difficulties attending classes or participating in office hours or on-campus tutorial services. Instructional support (tutoring, review sessions) and homework submission schedules are often impractical for commuter students at community colleges, where nearly half of all baccalaureate students start their college careers. In a report to the State University of New York [3], the Sloan Consortium points out that online courses are popular with students at two year colleges in New York who are not necessarily remote from campus, saying, “. . . our online courses are also very attractive to our traditional students, practicing teachers who often have responsibilities outside of the regular school day which make face-toface classes difficult to attend." An online learning component for existing courses in two-year colleges could have considerable impact on the success rate of students who are working adults traveling considerable distances.

Four-year colleges face a growing population of students needing remedial math, a drain on teaching and staff resources. From the student's perspective, the high cost of courses, textbooks and workbooks can be prohibitive. The high cost and inflexibility of proprietary software prevents many schools from providing an on-line option for interested students. Because Open Calculus is free, it may be used to prepare for a calculus course, reinforce student understanding during the course, and review material after the course is over. Because it is open source, institutions may customize a site to help students prepare for placement exams or other standardized tests. A site could also be adjusted to assist regional secondary school teachers by linking topics to state standards or the required textbook for a region.

The potential for Open Calculus to make a large-scale impact on student learning is thus great: Open Calculus can enable secondary schools with limited resources to offer calculus to their students; it can provide students at two-year colleges a range of resources that would otherwise be difficult for them to access; it can support remediation as well as regular instruction and review at four-year institutions. This paper describes what actually happened when this service was used in conjunction with calculus courses at two very different institutions, one a midsize four-year liberal arts college and the other a larger, urban two-year community college.

\section{HISTORY OF OPEN CALCULUS}

Open Calculus draws upon the work and products of several previous projects. It incorporates a collection of videos funded by the National Science Foundation (U.S.) as part of the Mathematics Across the Curriculum Project (1995-2002). It includes an extensive set of online problems, a well-developed WeBWorK prototype, and a collection of video tutorials whose development and evaluation were funded by a three-year "Cost Effective Uses of Technology" Mellon Foundation grant (1999-2001). Initial design of the online service took place in 2003 with support from the AT\&T Foundation. The Center for Cognitive and Educational Neuroscience at Dartmouth, supported by the National Science Foundation (U.S.), supported further development of practice problems and other materials for Open Calculus (2005-2006).

Although now intended to be a stand-alone service, Open Calculus originated in efforts to deepen the standard introductory calculus course at a liberal arts college. The development of online enhancements to a standard course was inspired by a wide-scale curricular shift in the United States that has become known as the "Reform Calculus" movement. Calculus reform was a reaction to earlier curricular revisions of the 1960's and 1970's that introduced a more robust mathematics sequence into high schools and colleges and moved students 
through it more quickly in an effort to produce more research scientists and mathematicians. This was not math for the masses, and by 1986, when a group of mathematicians initiated the calculus reform movement, national needs had changed sufficiently that the high attrition rate and lack of deep conceptual understanding among average students had become a matter of concern. Dubbing their reform "Toward a Lean and Lively Calculus," these mathematicians recommended new pedagogical approaches, new technology, and a new approach to content to improve conceptual understanding and help students apply what they had learned [4,5,6]. Answering the call for a calculus course that would be "a pump, not a filter," [7,8,9], faculty at the authors' institution set out to revitalize the introductory calculus course.

As part of this process, faculty identified a critical need to make the learning of routine calculus more efficient, so that all students could comfortably master regular course material. To that end, faculty developed a website to support the course, with a web page for each section and lecture that included practice problems, machine-graded homework problems, tutorial videos, and other resources. By 2002 the course included a new purpose-built text keyed to web pages for each section and lecture. By 2004 the web component had become sophisticated enough to become its own project, Open Calculus, and faculty at an urban two year institution in the western U.S. agreed to pilot the materials as part of a hybrid distance learning course. The term "hybrid" refers to the mix of traditional classroom work and web based resources that were used in this particular course.

\section{WHAT IS OPEN CALCULUS?}

Open Calculus is a set of online curricular tools, currently in development by the Dartmouth Mathematics Department, that aims to facilitate independent learning of calculus by providing online instruction (video and text), examples of worked-out problems, practice questions, and instant online grading of student answers to these questions. Online text and videos introduce and explain calculus concepts and work examples. The site is organized according to commonly recognized units of calculus learning (e.g., product and quotient rules, chain rule, exponential growth and decay), which can be keyed to a number of widely used calculus texts. Because the software and course is available online at no expense, it is accessible to schools and individuals regardless of their location or economic situation.

Open Calculus makes strong use of WeBWorK, an open source online (homework) problem delivery system developed at the University of Rochester with support from the Mellon Foundation, and currently maintained by and disseminated by Rochester. WeBWorK is a free automated homework system that generates unique problems for each student, provides them immediate right/wrong feedback and allows limitless trials to achieve the correct solution. Over 70 colleges and universities and a dozen high schools in the United States currently make use of WeBWorK software, making them natural candidates for hosting an Open Calculus server for their region [10]. WeBWorK received the 1999 International Conference on Technology in Collegiate Mathematics Award for Excellence and Innovation with the Use of Technology in Collegiate Mathematics.

WeBWorK was created with the dual goals of increasing the effectiveness of student learning and the efficiency of teaching, and their evaluation of its use indicates that it does both. As the developers point out on their web page [http://webwork.math.rochester.edu] (and as our evaluation results confirm), WeBWorK increases the effectiveness of student learning through traditional homework by providing immediate feedback about solutions so that students can correct mistakes while they are still thinking about the problem. WeBWorK comes with a large library of existing problems, but also allows individual instructors to author and display their own problems. Many institutions, including Dartmouth, use WeBWorK in conjunction with regular classes; we have extended its usability to anyone equipped with a browser, whether enrolled in a traditional calculus course or not.

\section{METHODOLOGY FOR EVALUATING THE ONLINE COMPONENTS OF OPEN CALCULUS}

This study evaluates the effectiveness of online components included in Open Calculus using data about student learning outcomes in courses at two very different institutions where those resources were employed. Thus we help to address a deficiency noted by Sunal et al [11], who conducted a meta-analysis of the research literature on best practices for developing online learning and concluded that "... despite the large volume of articles 
published at this time, most sampled include little discussion of the research design, data collection instruments or findings based on data gathered from course outcomes." We gathered data on student learning outcomes at a midsize liberal arts college where online resources were used to streamline the offering of the regular calculus course in order to make space in the curriculum for in-depth laboratory experiences. The online resources used were predecessors of the site we are now calling "Open Calculus" and were less complete. We also gathered data at a two-year college where a substantially revised version of the online materials was the basis for a "hybrid" distancelearning course.

At both institutions the effectiveness of the course with online components was evaluated against a control course lacking those components. The same evaluation instrument was used to measure student learning and attitude change at both institutions. Students in both the experimental and control courses completed a 35-question Likert-scaled math attitude survey on the first and last class day to measure change over the course interval in their confidence and perceived ability in mathematics, their math skills and study habits, their beliefs about the nature of mathematics and their attitudes toward different ways of teaching. Some items on the survey are phased so that the desired response is " $1=$ strongly agree" (e.g. "I am good at math.") and others are phrased so that the desired response is " 5 = strongly disagree" (e.g., "I try to avoid courses that involve mathematics."). For analysis the variables have been re-coded so that all desired responses have a value of " 5 " on the scale (thus desirable responses always have a higher value than undesirable ones, and pre-post changes in the desired direction are always positive).

The analysis of attitude change is based on surveys from those students in each class who completed both a pre- and post-survey. Due to late registrations and absenteeism, the number of matched surveys is always fewer than the total number of pre- and post-surveys, but the statistical advantages of using matched pairs more than offsets the loss in number.

To characterize pre-post change, a Survey Index for each individual and course was created by dividing the aggregate post-score by the aggregate pre-score and multiplying the result by 100 . An index of " 100 " thus shows no change; an index less than 100 shows change in the undesirable direction (the re-coded post-score/numerator was lower than the pre-score/denominator) and an index greater than 100 shows change in the desired direction (the recoded post-score was greater than the re-coded pre-score).

On the last class day students also assessed their own progress through a Learning Self-Assessment, indicating on a Likert-scaled form what gains in content learning, conceptual and contextual understanding, problem solving, and confidence they perceived as a result of their efforts in the class. Research has shown that student selfassessments concord highly with other measures of student achievement, making self-assessments a relatively reliable index of learning [12]. To characterize student learning gains, a Learning Self-Assessment Index was calculated by averaging all responses to the Learning Self-Assessment, dividing by the maximum score of " 5 " and multiplying by 100 . Thus a student with a mean Learning Self-Assessment score of 3.5 would have a Learning Self-Assessment Index of 70 ((3.5/5) x 100).

Finally, students at both institutions completed a short evaluation of the WeBWorK component of the course, assessing its usefulness in terms of motivation and learning.

It is important to note that the web-based materials used in these courses evolved considerably between the 2002 offering at the liberal arts college and the 2004 offering at the two-year community college. Early versions at the liberal arts college included applets and handwritten solutions to problems. The later version omitted these but had a wider range of practice problems and videos. For this and other reasons, it is not appropriate to compare the results at one institution with the results at the other one. Rather we consider the points of comparison to be with the standard course offerings that serve as control groups at each of these two institutions. Each experimental situation provides information about the efficacy of the online features. 


\section{The Liberal Arts College}

At the liberal arts college we compared the relative efficiency of a standard lecture version of the introductory calculus course held in 1999, which used computers only to facilitate computation for an additional laboratory component, with the technologically robust 2002 version of the same course. While the 2002 course continued to meet for lecture three times weekly, it was enhanced with web support including practice problems, machine-graded homework problems, instructional videos, interactive applets, hand-written problem solutions, an online text and other features. This approach thus compares outcomes longitudinally rather than contemporaneously, a strategy necessitated by the lack of a comparable contemporaneous course but fully justified by the consistency of instruction and student population from year to year. The same instructor taught the course each year. Students were virtually all first-year students and analysis of the population in terms of gender, ethnicity, anticipated major, preparation (as measured by incoming SAT scores) and math attitudes (as measured on the attitude pre-survey) reveals no differences in the student cohorts from year to year.

The research used multiple data sources to provide a balanced picture of student experience. In addition to the pre-post attitude survey and learning self-assessment described above, in-depth student interviews were conducted following the 2002 iteration. Interview data contextualize and expand survey results. A random sample of students (about 10\% of the two hundred enrolled) were interviewed in the early weeks of the term following the 2002 offering, when they talked in greater depth about their own background, their academic intentions, and their response to the course. In-depth student interviews were not conducted in 1999, but an existing corpus of over 70 interviews from 1997 and 1998, when the course was also under careful evaluation, allows us to interpret the 1999 survey responses adequately. At the end of the course students also completed a short evaluation of the WeBWorK component.

\section{The Two-Year Community College}

In Fall 2004 the Open Calculus project was piloted at a two-year urban school of about 11,000 students. At this community college, the Open Calculus web site was used to supplement one of two sections of a first-term calculus course, making it a "hybrid" section in the institution's designation, while the unmodified (standard) section served as the control. As at the liberal arts college, students in the experimental and control courses at the community college were demographically very similar. A review of pre-surveys completed by 27 students in the hybrid course and 22 in the standard course shows that students in both courses were young (90\% were under 25) and male (at least two-thirds of each class were men). There were no differences in terms of ethnicity or mathematics preparation; about a quarter of each class had completed a college algebra course, and about half had taken two terms of pre-calculus. Most were science or business majors. But there were some telling differences on the attitude pre-survey. Students in the standard class were significantly more likely to believe that using a computer makes mathematics more complicated than it needs to be, suggesting that each course attracted students who preferred its pedagogy.

In the hybrid course, WeBWorK homework accessed through Open Calculus was the only graded homework, although some problems from the textbook were assigned for more practice. The Open Calculus videos were shown in class to provide additional examples of problem solutions. Examinations were given in class, although in the hybrid some quizzes were administered online through commercial software. In the standard course the commercial software was used only to post worksheets and homework assignments. During the first week, students in the hybrid course were provided a short in-class introduction to the commercial software, the Open Calculus site, and the WeBWorK site. There were no special tutorials or other assistance to help students navigate the electronic resources. These were the only features that distinguished the hybrid section from the standard. In both, lecture/discussion was the primary way of presenting material. A different experienced full-time faculty member taught each section. The hybrid section was clearly marked as such in the course listings and students chose which section they desired; they were not randomly assigned. 
In addition to the attitude survey and learning self-assessment, students in the hybrid class also completed a short evaluation of the WeBWorK homework system. This report uses the results of these evaluation instruments to compare the outcomes of the two sections.

\section{RESULTS}

\section{Overview}

At the liberal arts college, students using the online system as a supplement to class lecture scored better than those in the lecture-only course on measures of content learning, mathematics attitudes and skills. At the community college, learning and attitude outcomes were about the same, but students using the online resources achieved those results in $80 \%$ of the time required by students in the standard course. Extensive interview and survey data show that students strongly endorse the WeBWorK system that provides immediate right/wrong feedback and allows limitless trials to achieve the correct solution. WeBWorK motivated students to complete homework regularly and immediately expunged incorrect approaches from their repertoires, making learning more efficient. Additionally, WeBWorK's automation of the collection and grading of all homework resulted in cost savings for the institutions. Students also valued the short videos, which provided a reference in a different medium-and hence perspective-from the text, but which, like the text, could be consulted at any time and as often as necessary.

\section{The Liberal Arts College}

Learning self-assessments, survey results, and interviews all indicated that the new online features helped students learn calculus better. On the Student Learning Self-Assessment, students rated their gains, as a result of taking this course, in thirteen different areas. Using factor analysis we have constructed four Self-Assessment outcome indices: the Content Index (averaging self-assessed learning in four areas: elementary functions, derivatives, integrals, and modeling with differential equations), the Thinking Index (conceptual understanding and problem-solving skills), the Relationships Index (understanding how calculus relates to other disciplines and realworld issues), and the Comfort Index (personal comfort and enthusiasm for mathematics). We also constructed an Overall Learning Self-Assessment Index averaging all items. In each case, student scores are expressed as a fraction of the highest possible score and multiplied by 100. Table 1, which compares the Learning Self-Assessment scores for 1999 and 2002, shows that students using the online resources felt they learned more than students in the standard course.

Table 1. Learning Self-Assessment Index Scores, Liberal Arts College

\begin{tabular}{|c|c|c|}
\hline Index & $\mathbf{1 9 9 9}$ & $\mathbf{2 0 0 2}$ \\
\hline Content & 75 & 78 \\
\hline Thinking & 64 & 69 \\
\hline Relationships & 67 & 67 \\
\hline Comfort & 49 & 56 \\
\hline Overall & $\mathbf{6 5}$ & $\mathbf{6 8}$ \\
\hline
\end{tabular}

Students in the 2002 online course also emerged with the more strongly desirable beliefs and attitudes about mathematics than in previous years. The Mathematics Survey measures change between the first and last class day in students' assessments of their mathematical ability, their interest in mathematics, and their appreciation for the relevance of mathematics to the real world and to their own lives. It also asks them how they view the use of computers to do mathematics. The Survey Index, which characterizes the pre-post attitude change as a whole, stood at 97.5 for 2002; for 1999 it was 96.2. Students in the course with online resources thus reported more desirable mathematics attitudes as a result of their calculus experience than did students in the standard course. 
It is, of course, the goal of this (or any) calculus course that students leave feeling more able and interested in mathematics and with a greater appreciation of its relevance. This is a good place, therefore, to comment on the absolute value of the index, which for this introductory calculus course has never exceeded 100, the level of "no change." Most students who take this course are first-year first-term students, and at this college introductory calculus is typically the most difficult course they encounter, moving much faster than their high school course (where most did well) and requiring more independence in learning. Thus in some certain — but indefinable — way, the attitude scores reflect the difficulties in the overall transition to college many students are experiencing and their irritation at what they may perceive as the "extra" work of the laboratory exercises.

It is also worth noting that the addition of online components appears to benefit science majors more than others. This calculus course has always been aimed at prospective science majors who need a solid mathematical base in order to initiate a science sequence, but many non-science majors also enroll. Different pedagogical approaches call upon different student skills and aptitudes and tend to advantage different sub-populations of students. While the non-science majors did not suffer in the transition from a lecture to an online-supported course, neither did they gain. Their scores remain virtually unchanged between 1999 and 2002 . Science students, on the other hand, posted an index increases of $8 \%$ between 1999 and 2002. It would appear that the online resources improved the learning of the target population without disadvantaging others.

The broad range of online resources in the 2002 course offering, including the WeBWorK homework system, interactive applets illustrating concepts, practice problems and exams, video tutorials, and interesting links, were all designed to support mathematics learning. Student interviews suggest that students saw this array as a kit of tools to be accessed as needed. Some students reported that the only online resource they used was WeBWorK, through which they submitted required homework. Others availed themselves of the full complement. When asked to explain why they didn't use a resource, the answer was always the same: "I didn't need it." Students are very pragmatic; their goal is to understand the material well enough to get the grade they desire, and while they are aggressive in pursuing that goal, they stop when their understanding feels secure. As one explained, "I didn't really explore the web site. I just used what I needed."

Some students needed only lectures and homework to achieve understanding. Others used the applets to help them visualize concepts, worked and reviewed many practice problems, and viewed video tutorials. There were 650 "hits" on the hand-written problem solutions during the ten-week term, mostly in October and November, suggesting that these examples were useful to many. The fact that some students rarely used the applets or never looked at a video clip does not mean that these were not valuable resources for the course, anymore than the fact that most citizens don't use the welfare system means it should not be in place. This introductory class attracts students with a wide range of backgrounds and levels of confidence, and the goal of the course is that they all master the material. The question is not simply how widely used the resources were, but whether those who needed and used them found them helpful. Overwhelmingly, they did. Consider this student's comment.

I would try to go through the problem by myself a couple of different ways but if I just wasn't getting the answer-if it wasn't the right answer for the WeBWorK-I would finally go to view the video and then after going through the video a couple of times and watching how the problem was set up and carried out then I would apply that to the problem that we had and then most of the time I would do okay.

Most of the resources were used on an "as needed" basis, but all students had to submit homework using WeBWorK. Like most mathematical software, WeBWorK requires answers to be submitted in a specified syntax. Improperly represented answers, even if substantively correct, are rejected as incorrect. This rigidity was unfailingly frustrating to students, but it was more than offset by the system's benefits: WeBWorK was otherwise easy to use, portable, provided "right/wrong" feedback, and allowed an unlimited number of attempts to get the right answer. Students seem to appreciate, as educators already know, how important it is to expunge incorrect information quickly and replace it with the correct understanding. On the WeBWorK evaluation completed on the last class day, $85 \%$ of 2002 students said they liked WeBWorK's immediate feedback feature and $60 \%$ said it motivated them to persist more with the assignments. 
Student interviews emphasize the importance of immediate feedback in terms of learning and motivation, and help to put the "irritation" factor in perspective. Overall, nine of the interviewees endorsed the system, two did not, and two were equivocal. Taken as a whole, students valued the convenience and information of the online features. As one summarized, "I would say we have a pretty good web site. It is very helpful. It has more things than I need." This student comment is typical.

I think I actually like it better than handing in paper copy because it lets you know you have the wrong the answer and you have another chance to figure it out. And so for me I like to try things different ways, because I know, especially in the math world, one way at getting there is not always the best way of getting there. So it tells me that quick. The problem with WeBWorK is getting to know the notations that it accepts, but once you get past that, once you get used to it, I don't think it's that complicated.

Taken together, the improvements in the survey measures, although not statistically significant, and the student interview comments suggest that the addition of online features improved student learning in the course. No category of student was disadvantaged by the switch to an online format and, furthermore, the science students who are the target audience for the course realized notable gains.

\section{The Two-Year Community College}

The Learning Self-Assessment for the community college was modified slightly to fit the content and aims of the course. It therefore included only three content-learning items, but added two items about work habits not included in the instrument used at the liberal arts college. This change resulted in slightly different outcome indices, but does not affect the evaluative utility of the instrument, since the only within-institution comparisons are valid for our purposes. Factor analysis produced the following four indices: the Content Index (averaging self-assessed learning in three areas: elementary functions, derivatives and integrals), the Thinking/Problem-solving Index (conceptual understanding and problem-solving skills), the Work Habits Index (working effectively alone and in a group), and the Connections/Confidence Index (understanding how calculus connects to other disciplines and realworld issues and enthusiasm and comfort regarding math). Table 2 shows that both courses conveyed the mathematics content well, but overall the standard course was slightly more successful in instilling skills and positive attitudes.

Table 2. Learning Index Scores, Community College

\begin{tabular}{|c|c|c|}
\hline Index & Standard & Hybrid \\
\hline Content & 80 & 80 \\
\hline Thinking/problem-solving & 74 & 72 \\
\hline Work habits & 64 & 70 \\
\hline Connections/confidence & 68 & 64 \\
\hline OVERALL & $\mathbf{7 0}$ & $\mathbf{6 8}$ \\
\hline
\end{tabular}

On the other hand, the attitude survey results show that the hybrid course appears to have been somewhat more successful than the standard in improving student attitudes about mathematics. Comparing the pre-post responses to the 35 items on the attitude survey, the hybrid course had an overall Survey Index of 111 and the comparison course had a Survey Index of 103. Looking at individual items, there was no clear pattern of strength or weakness in either course and, not surprising in such a small sample, there were no significant differences between them on any variable. Both sections maintained or improved student attitudes across the board, with the hybrid course showing change in the desired direction on 30 of 35 variables and the standard course on 32 of 35 . Neither the Attitude Survey difference nor that recorded on the Learning Self-Assessment is statistically significant in this small population. Recalling that these populations are self-selected, with students choosing the pedagogy they preferred, we conclude that each served its students equally well. 
The hybrid course, however, appears to be more efficient. Students in both courses felt they learned "a lot" about the material itself and also made good progress in their conceptual understanding, problem-solving skills, work habits, understanding the relevance of calculus, and math confidence and interest. What is different is how long it took them to accomplish that. Students in the standard course spent 36\% longer doing homework than those in the hybrid course and $26 \%$ longer altogether working on calculus to achieve the same results. (Again, since this is a retrospective question, like the learning self-assessment, we have considered all available data). Although the difference is not statistically significant, owing the small population, these data suggest that the online environment provides a more efficient route to calculus learning than the standard paper and pencil homework approach. Students in the hybrid course also were significantly more likely to complete their homework on time and to understand that being good at math does not necessarily meaning doing math quickly.

Community college students' evaluation of WeBWorK tells us something about why WeBWorK may have provided greater efficiency. Students overwhelmingly endorsed WeBWorK's instant feedback feature, and a strong majority felt that they learned from WeBWorK, that it offered good preparation for exams and that it encouraged them to persist. Few indicated ongoing problems with access to computers or with the syntax, two potential stumbling blocks for usage. $60 \%$ preferred WeBWorK to paper and pencil, and $65 \%$ gave the online homework system a "thumbs up" overall.

\section{CONCLUSIONS}

\section{The Liberal Arts College}

All the evaluation measures indicate that the 2002 offering is a better course than its 1999 predecessor. Students in the 2002 course rated their mathematics learning, and their acquisition of allied skills and attitudes, higher than in the unmodified 1999 baseline course. Their attitudes and beliefs about mathematics, which typically decline during the first college math course, showed the smallest erosion in the four years between the baseline and target course. They reported that they learned more from the laboratory exercises, and with the least investment of effort, than ever before. The role of the online resources in promoting learning is clear. More than in past years, students believe that using the automated WeBWorK helped them learn the math, and they value computers as an aid to doing mathematics. Since computers are inescapable in contemporary business and research, increasing students' appreciation for their role in problem solving is a valuable contribution in itself. Interview data suggest that students are comfortable with the online format and value its interactive qualities, especially the immediate feedback from WeBWorK, which prevents them from pursuing unproductive learning paths. Students appear to access the online resources on as "as needed" basis, with some students using only the required WeBWorK, while others employed the full range in order to understand the calculus. This seems appropriate and, on reflection, expectable. Students have always behaved this way-some never attended office hours or tutorials, others frequently did - and instructors have always organized course support with the needier students in mind. The fact that few students watched the video tutorials, for example, is less important to our assessment of their value than the fact that those who did so found them helpful.

The more efficient use of instructor time may also contribute to increased student learning. The automated grading process meant that in 2002 instructors at all levels could devote a much higher proportion of their time to tutorial activities. While the number of people involved in teaching the course dropped from fifteen in 1999 to nine in 2002, there was an increase in the total number of student-contact hours. It seems reasonable to suppose that the increased time spent by faculty and student assistants helping students directly contributed to the increased learning.

\section{The Two-Year Institution}

The survey of attitude change and students' self-evaluation of their learning revealed that the hybrid and standard classes were highly similar in outcome. Students emerged from both sections with more positive attitudes about mathematics overall and with strong self-perceived learning gains. The introduction of an online homework system and the viewing of calculus videos does not seem to have advantaged — or disadvantaged — the students in the hybrid course in either regard. What it does appear to have offered them is a more efficient mechanism for 
learning. The student populations are small here, and all conclusions must be regarded as provisional, but students in the hybrid course spent less time on homework (3.5 hours per week less) and on the course overall (4.4 hours per week less) than students in the standard course.

\section{SUMMARY}

Outcomes at the liberal arts college and the community college confirm the potential of Open Calculus to improve learning for students. Evaluation of elements which are central to Open Calculus in these two diverse settings suggest that online resources, especially the WeBWorK online homework system, help students learn more efficiently and, in classroom settings like these, give faculty more time for direct interactions with students. Because Open Calculus is free and open source, it can be installed at any institution and modified to meet the requirements of any course.

Open Calculus is available for use now at http://www.math.dartmouth.edu/opencalc2/. For further information about Open Calculus, please contact the Open Calculus Project at opencalc@ math.dartmouth.edu.

\section{DEDICATION}

This paper is dedicated to the memory of Donald Kreider (1931-2006), Professor of Mathematics at Dartmouth College and colleague, mentor and friend to all three authors.

\section{ACKNOWLEDGEMENETS}

This work has been supported by NSF grants \#94552462 [MATC] and $0354400\{C C E N\}$ by a Mellon Foundation "Cost-Effective Uses of Technology in Teaching" grant, and by and AT\&T Foundation grant "Development and Dissemination of a Free Online Calculus Course".

\section{REFERENCES}

1. Anaya, G. (1999) College impact on student learning: Comparing the use of self-reported gains, standardized test scores and college grades. Research in Higher Education 40, 499 - 526.

2. Bookman, J. \& Friedman, C. P. (1994). A comparison of the problem solving performance of students in lab based and traditional calculus. In E. Dubinsky, A. H. Schoenfeld, \& J Kaput, (Eds.), Research in Collegiate Mathematics Education. I. Providence, R. I. : American Mathematical Society.

3. Douglas, R. G., Ed. (1986). Toward a Lean and Lively Calculus. Washington, D.C.: Mathematical Association of America.

4. Factors Influencing Faculty Satisfaction with Asynchronous Teaching and Learning in the SUNY Learning Network, A report to SUNY by the Sloan Consortium, http://www.sloanc.org/consulting/docu/sc/59/120.rtf.

5. Hurley, J. F., Koehn, U. \& Ganter S. L. (1999). Effects of Calculus Reform: Local and National. American Mathematical Monthly 106: 800 - 811.

6. National Center for Educational Statistics, http://nces.ed.gov/programs/digest/d02/dt419.asp

7. National Rural Education Association, http://www.nrea.net/

8. Steen, L., Ed. (1988). Calculus for a New Century. Washington, D.C.: Mathematical Association of America.

9. Sunal, Sunal, Odell and Sundberg, Research-Supported Best Practices for Developing Online Learning, Journal of Interactive Online Learning Volume 2, Number 1, Summer 2003

10. Tucker, A. C. \& Leitzel, J. R. (1995). Assessing Calculus Reform Efforts: A Report to the Community. Washington, D. C. : The Mathematical Association of America.

11. UME Trends, Vol 6, No.6 (January 1995). Special Issue on Calculus Reform.

12. University of Rochester WeBWorK User Site, http://math.webwork.rochester.edu/docs/sites/courses/links_to_courses.html 